\title{
NOUVELle
}

\section{La myéline}

\section{Un nouvel acteur dans la plasticité cérébrale}

Lucas Baudouin, Noémie Adès, Lamia Bouslama-Oueghlani

Sorbonne Université, Inserm U1127, CNRS UMR7225, Institut du cerveau-ICM, 47, boulevard de l'Hôpital, 75013 Paris, France. lamia.bouslama@sorbonne-universite.fr
> Dans le système nerveux central (SNC), la «plasticité » a longtemps été associée uniquement aux neurones. La plasticité neuronale désigne en effet la capacité des réseaux de neurones à moduler leur fonctionnement, à se sensibiliser ou à se désensibiliser face à un stimulus, à créer de nouvelles synapses ou à en supprimer. Cette plasticité synaptique permet notamment l'apprentissage et l'adaptation de l'individu à son environnement. Des articles publiés au cours des dernières décennies ont cependant montré qu'il existait également une plasticité de la myéline, une structure multi-lamellaire isolant les axones ${ }^{1}$ et considérée auparavant comme statique.

\section{La plasticité de la myéline}

Chez l'homme, l'imagerie par résonance magnétique (IRM) a révélé l'existence de changements de la «substance blanche » (un indicateur de la myélinisation des axones) en fonction de l'expérience (Figure 1). Ainsi, la pratique du piano, du jonglage, ou encore l'apprentissage de la lecture ou de nouvelles langues entraînent une augmentation du volume de la substance blanche [1, 2]. Inversement, une altération des faisceaux de la substance blanche reliés au cortex préfrontal a été observée chez des enfants élevés dans des institutions où ils étaient maltraités [3]. Bien que I'IRM ne permette pas de visualiser spécifiquement la myéline, l'utilisation de

1 L'axone, ou fibre nerveuse, est le prolongement du neurone qui conduit le signal électrique du corps cellulaire vers la zone synaptique. modèles animaux, quant à elle, a permis de démontrer qu'il existe en effet une plasticité de la myéline, appelée myélinisation adaptative.

Le circuit neuronal impliqué dans l'audition binaurale, qui permet de localiser une source sonore, fournit un autre exemple des capacités adaptatives de la myélinisation [4]. En effet, une myélinisation différentielle entre les branches ipsi-latérale et contra-latérale des neurones du noyau cochléaire du tronc cérébral a été observée. Ce profil différentiel de myélinisation permet un acheminement plus rapide du signal électrique dans les branches contra-latérales plus myélinisées, ce qui, dans cet exemple, compense le trajet plus long emprunté par ces dernières. La myélinisation des fibres nerveuses, dont dépend la vitesse de conduction de l'influx nerveux, a donc un effet majeur sur la réponse synaptique, en permettant par exemple de coordonner les temps d'arrivée des signaux électriques de différents neurones à une même cible postsynaptique afin de maximiser la sommation temporelle. Par conséquent, une myélinisation adaptative permet également de répondre à certaines nécessités physiologiques de fonctionnement des circuits neuronaux.

La myélinisation adaptative concerne non seulement le cerveau en développement, mais aussi le cerveau mature, dans lequel des cellules précurseurs des oligodendrocytes (oligodendrocyte precursor cells, OPC), nombreuses (elles représentent 2 à $9 \%$ des cellules du SNC [5]), sont capables de proliférer, de se différencier en oligodendrocytes et de former de nouvelles gaines de myéline autour des axones [6-9]. Les axones des neurones du SNC ne sont pas tous complètement myélinisés : certains axones sont myélinisés de façon discontinue et ont ainsi des fragments dépourvus de myéline $[8,9]$, tandis que d'autres ne sont pas du tout myélinisés. Dans le corps calleux d'un cerveau de rongeur adulte, seulement $30 \%$ des axones sont myélinisés [10]. Enfin, il est intéressant de noter que les OPC forment des synapses (glutamatergiques ou GABAergiques) avec des neurones provenant de différentes régions [11]. Par conséquent, les OPC pourraient répondre aux changements de l'activité électrique de différents circuits neuronaux.

Ainsi, les OPC du cerveau mature participent non seulement à la régénération de la myéline dans les lésions de démyélinisation dans le cas de maladies comme la sclérose en plaques, mais aussi à la formation de nouveaux oligodendrocytes nécessaires à la myélinisation adaptative dans le SNC sain. La grande stabilité des oligodendrocytes nouvellement générés, suivis par traçage génétique dans différentes régions du SNC, est une preuve supplémentaire de leur rôle adaptatif [12]. Cependant, dans le nerf optique, où quasiment tous les axones sont myélinisés, cette production d'oligodendrocytes semble participer à un renouvellement des gaines de myéline, plutôt qu'à la synthèse de gaines sur des portions d'axones non myélinisées [6, 12].

\section{Comment la plasticité myélinique est-} elle contrôlée?

Le changement du profil de myélinisation pourrait s'opérer par : 1) la myélinisation 


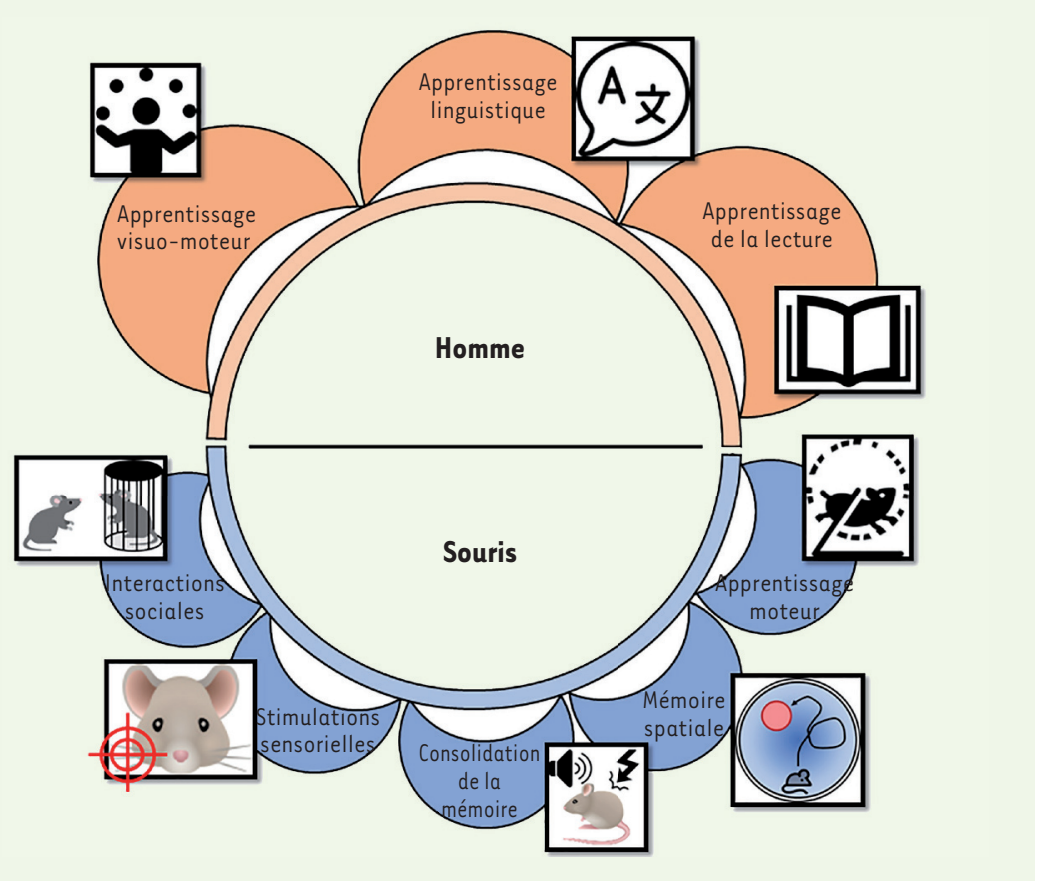

Figure 1. L'expérience et l'apprentissage modifient la structure de la substance blanche et le profil de myélinisation dans le cerveau mature. Dans l'espèce humaine, des changements structurels de la substance blanche ont lieu lors de l'apprentissage de tâches motrices complexes, lors de l'apprentissage de nouvelles langues, ou encore lors de l'apprentissage de la lecture. Dans des modèles murins, il a été montré que la privation d'interactions sociales provoque une hypomyélinisation ciblée dans le cortex préfrontal. A contrario, la stimulation sensorielle des vibrisses, la consolidation ou le rappel de la mémoire de la peur, ou encore la mémoire spatiale et l'apprentissage moteur changent le profil de myélinisation (sources des images : https://icones8.fr).

des axones non myélinisés ; 2) la myélinisation de segments d'axones partiellement myélinisés; 3) l'augmentation ou la réduction de la longueur et de l'épaisseur des segments myéliniques entre les nœuds de Ranvier² (segments internoeuds) des axones myélinisés; 4) enfin, le changement de la longueur des nœuds de Ranvier (Figure 2). Toutes ces modifications sont susceptibles de changer le fonctionnement des circuits neuronaux en modifiant la vitesse de conduction de l'influx nerveux et le support métabolique des neurones [13].

\footnotetext{
2 Dans les axones myélinisés, les nœuds de Ranvier (du nom de leur découvreur, Louis-Antoine Ranvier [1835-1922]) désignent de très courts segments de la membrane axonale dépourvus de la gaine de myéline électriquement isolante. La présence des nœuds de Ranvier échelonnés le long de l'axone permet la conduction saltatoire, de nœud en nœud, d'un influx nerveux (potentiel d'action).
}

des souris. De même, l'apprentissage d'une tâche sensitivo-motrice chez des rats, comme celle de saisir une boule de sucrose avec la patte avant droite, entraîne une augmentation de la myélinisation dans la substance blanche située au-dessous de la région du cortex moteur gauche qui contrôle la motricité de cette patte [14]. De plus, la production de nouveaux oligodendrocytes est nécessaire pour l'apprentissage de nouvelles tâches motrices. La déplétion, spécifiquement dans les OPC de souris adultes, d'un facteur régulateur de la transcription (myelin regulatory factor, MYRF) essentiel pour la maturation des oligodendrocytes et l'intégrité de la myéline, inhibe l'apprentissage moteur $[15,16]$. Plus récemment, trois groupes de recherche ont montré que les oligodendrocytes et la myéline nouvellement produits chez la souris adulte contribuent à l'acquisition et à la consolidation de la mémoire [17]. Des expériences réalisées par un de ces groupes ont montré que l'inhibition de la production de nouveaux oligodendrocytes et de myéline entraîne des déficits de la mémoire spatiale chez des souris jeunes, alors que sa stimulation corrige ce défaut chez des souris âgées, dont la mémoire décline au cours du vieillissement [17]. A contrario, certaines situations, telles que la privation sensorielle, induisent une diminution de la myélinisation. L'isolement social provoque une hypomyélinisation spécifique du cortex préfrontal et des déficits dans les interactions sociales, que l'isolement soit réalisé pendant la période juvénile ou à l'âge adulte [18, 19]. Par ailleurs, une thérapie pro-myélinisante chez les souris adultes isolées corrige ces défauts [18], ce qui témoigne encore de l'importance de la myélinisation adaptative.

Si ces études ont permis d'asseoir le concept de la plasticité de la myéline chez la souris, la visualisation de cette plasticité en temps réel reste un défi, que les avancées majeures de l'imagerie longitudinale par microscopie optique à fluorescence chez la souris vivante 


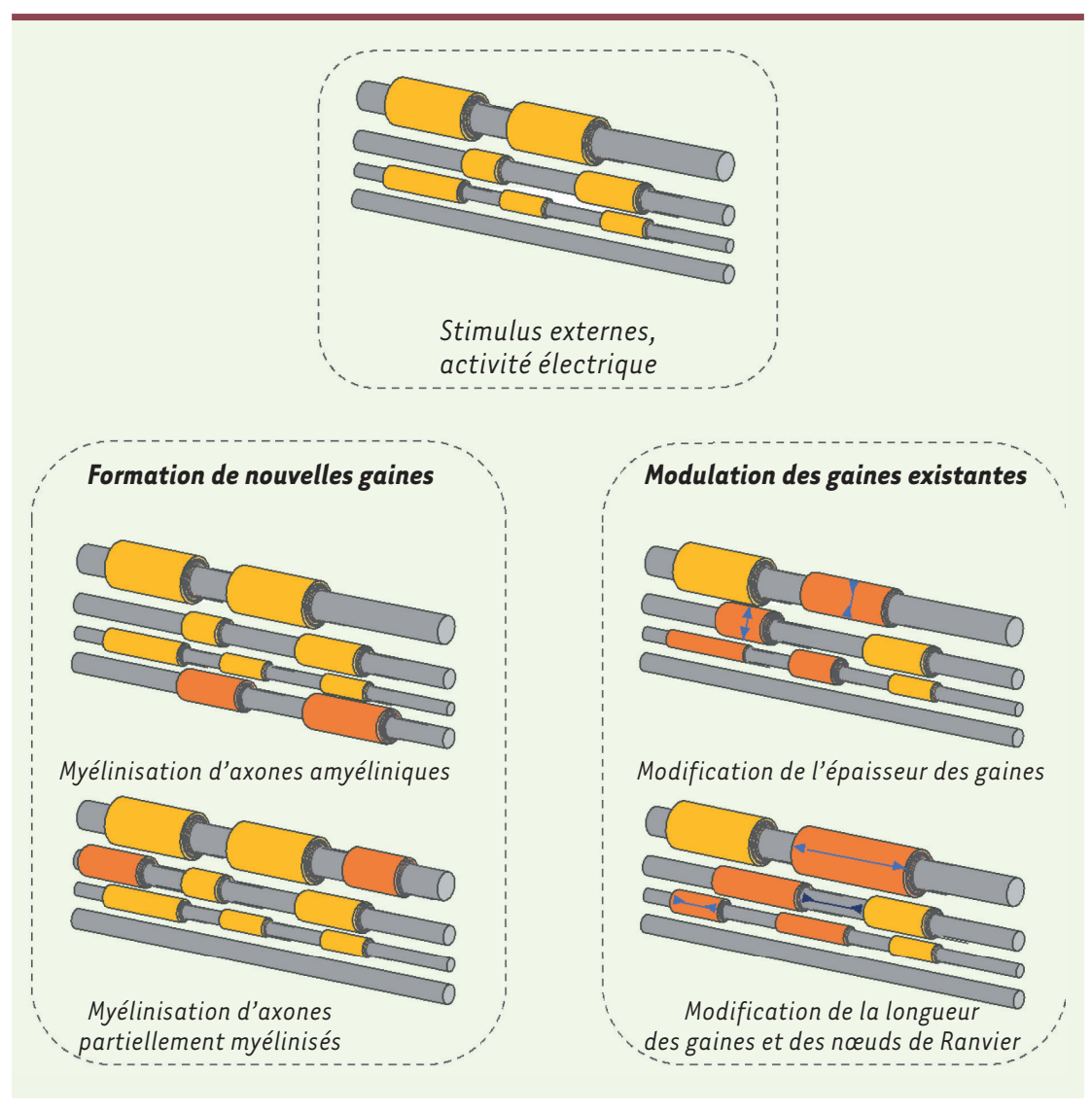

ont permis de relever. En effet, deux études récentes ont montré que de nouveaux segments de myéline sont continuellement produits dans les couches superficielles du cortex somato-sensoriel de la souris adulte $[8,9]$. En analysant la dynamique de segments myéliniques pendant plusieurs mois, chez des souris âgées de 2-3 mois, il a été montré que certains subissent une modification de leur longueur : $15 \%$ d'entre eux subissent une extension et $4 \%$ une rétraction, tandis que la grande majorité de ces segments ( $81 \%$ ) restent stables [9]. La même étude réalisée chez des souris âgées d'un an montre que la modification de longueur (extension ou rétraction) concerne moins de $1 \%$ des segments myéliniques [8], une différence que pourrait expliquer la diminution du nombre d'axones partiellement myélinisés entre l'âge de trois mois et l'âge d'un an [9]. Néanmoins, même si d'après ces résultats, le vieillissement ne semble pas propice à la plasticité
Figure 2. Différentes formes de plasticité myélinique. En fonction des stimulus environnementaux ou de l'activité neuronale, le profil de myélinisation évolue: (1) de nouvelles gaines de myéline peuvent être formées autour d'axones non ou partiellement myélinisés; (2) la longueur et l'épaisseur des gaines préexistantes, ainsi que l'architecture des nœuds de Ranvier peuvent être modifiées.

hypothèse, la synthèse de nouveaux oligodendrocytes au cours de l'apprentissage moteur, chez la souris, a lieu pendant la période qui correspond à celle de la consolidation synaptique [16]. En outre, une étude récente a montré que la stimulation de la myélinisation chez des souris âgées empêche la perte des synapses dans l'hippocampe et améliore ainsi la mémoire spatiale [17]. Cependant, les mécanismes moléculaires qui aboutissent à la myélinisation adaptative restent à élucider. La modulation du cytosquelette d'actine, très fortement impliqué dans la myélinisation, pourrait jouer un rôle central dans la plasticité myélinique, comme c'est d'ailleurs le cas pour la plasticité synaptique [1]. $\diamond$

Myelin: A new player in brain plasticity

\section{REMERCIEMENTS}

Nous remercions la fondation pour la recherche contre la sclérose en plaques (ARSEP) ainsi que la fondation Marie-Ange Bouvet Labruyère pour leur soutien financier. Nous remercions également Brahim Nait Oumesmar et Nicolas Brard pour la relecture du manuscrit.

\section{LIENS D'INTÉRÊT}

Les auteurs déclarent n'avoir aucun lien d'intérêt concernant les données publiées dans cet article.

\section{Perspectives}

La plasticité de la myéline englobe des modifications de sa structure et de sa densité. Ainsi, tout comme la plasticité synaptique, la plasticité myélinique est modulée par des stimulus externes. Dès lors, la plasticité des circuits cérébraux pourrait dépendre d'une interdépendance étroite des plasticités myélinique et synaptique. En faveur de cette

\section{RÉFÉRENCES}

1. Stadelmann C, Timmler S, Barrantes-Freer A, Simons M. Myelin in the central nervous system: structure, function, and pathology. Physiol Rev 2019 ; 99 : 1381-431.

2. Fields RD. A new mechanism of nervous system plasticity: activity-dependent myelination. Nat Rev Neurosci 2015 ; 16 : 756-67. Neurodevelopmental effects of early deprivation in postinstitutionalized children. Child Dev $2010 ; 81$ : 224-36.
3. Pollak SD, Nelson CA, Schlaak MF, et al. 


\section{RÉFÉRENCES}

4. Seidl AH. Regulation of conduction time along axons. Neuroscience 2014 ; 276 : 126-34.

5. Boshans LL, Sherafat A, Nishiyama A. The effects of developmental and current niches on oligodendrocyte precursor dynamics and fate. Neurosci Lett $2020 ; 715$ : 134593.

6. Young KM, Psachoulia K, Tripathi RB, et al. Oligodendrocyte dynamics in the healthy adult CNS: evidence for myelin remodeling. Neuron 2013 ; 77 : 873-85.

7. Gibson EM, Purger D, Mount CW, et al. Neuronal activity promotes oligodendrogenesis and adaptive myelination in the mammalian brain. Science 2014 $344: 1252304$.

8. Hughes $\varepsilon G$, Orthmann-Murphy JL, Langseth AJ, Bergles DE. Myelin remodeling through experience-dependent oligodendrogenesis in the adult somatosensory cortex. Nat Neurosci 2018 ; 21 : 696-706.
9. Hill RA, Li AM, Grutzendler J. Lifelong cortical myelin plasticity and age-related degeneration in the live mammalian brain. Nat Neurosci 2018 ; 21 : 683-95.

10. Sturrock RR. Myelination of the mouse corpus callosum. Neuropathol Appl Neurobiol $1980 ; 6: 415-20$.

11. Habermacher C, Angulo MC, Benamer N. Glutamate versus GABA in neuron-oligodendroglia communication. Glia 2019; 6 : 2092-106.

12. Tripathi RB, Jackiewicz M, McKenzie IA, et al. Remarkable stability of myelinating oligodendrocytes in mice. Cell Rep $2017 ; 21: 316-23$.

13. de Faria 0 Jr., Pama EAC, Evans K, et al. Neuroglial interactions underpinning myelin plasticity. Dev Neurobiol 2018 ; 78 : 93-107.

14. Sampaio-Baptista C, Khrapitchev AA, Foxley S, et al. Motor skill learning induces changes in white matte microstructure and myelination. J Neurosci 2013 ; 33 : 19499-503.

15. McKenzie IA, Ohayon D, Li H, et al. Motor skill learning requires active central myelination. Science 2014 ; $346: 318-22$.
16. Xiao L, Ohayon D, McKenzie IA, et al. Rapid production of new oligodendrocytes is required in the earliest stages of motor-skill learning. Nat Neurosci 2016 ; 19 1210-7.

17. Xin W, Chan JR. Myelin plasticity: sculpting circuits in learning and memory. Nat Rev Neurosci 2020.

18. Liu J, Dupree JL, Gacias M, et al. Clemastine enhances myelination in the prefrontal cortex and rescues behavioral changes in socially isolated mice. J Neurosci 2016 ; 36 : 957-62.

19. Makinodan M, Rosen KM, Ito S, Corfas G. A critical period for social experience-dependent oligodendrocyte maturation and myelination. Science 2012 ; 337 : 1357-60. 